\title{
Low Levels of Gastrin 17 are Related with Endoscopic Findings of Esophagitis and Typical Symptoms of GERD
}

Francesco di Mario ${ }^{1}$, Pellegrino Crafa ${ }^{2}$, Marilisa Franceschi $^{3}$, Kryssia Isabel Rodriguez-Castro ${ }^{3}$, Gianluca Baldassarre ${ }^{3}$, Antonio Ferronato $^{3}$, Antonio Antico ${ }^{4}$, Maria Piera Panozzo ${ }^{4}$, Lorella Franzoni ${ }^{1}$, Alberto Barchi ${ }^{1}$, Michele Russo ${ }^{1}$, Nicola de Bortoli ${ }^{5}$, Matteo Ghisa ${ }^{6}$, Edoardo Savarino ${ }^{6}$

1) Gastroenterology Unit,

Department of Medicine and Surgery, University of Parma, Parma;

2) Pathology Unit, Department of Medicine and Surgery, University of Parma, Parma;

3) Endoscopy Unit,

Department of Medicine, ULSS7 Pedemontana, Hospital AltoVicentino, Santorso (VI);

4) Laboratory of Clinical Pathology, ULSS7

Pedemontana, Hospital AltoVicentino, Santorso (VI); 5) Department of Translational Research and New Technology in Medicine and Surgery, Division of Gastroenterology, University of Pisa, Cisanello Hospital, Pisa;

6) Gastroenterology

Unit, Department of Surgery, Oncology and Gastroenterology, University of Padua, Padua, Italy

\author{
Address for correspondence: \\ Francesco Di Mario, MD, \\ Department of Medicine and \\ Surgery, University of Parma, \\ Via Gramsci, n. 14, 43126, \\ Parma, Italy, \\ francesco.dimario@unipr.it
}

Received: 25.08.2020 Accepted: 13.11.2020

\section{ABSTRACT}

Background \& Aims: In clinical practice, most patients with symptoms suggestive of gastroesophageal reflux disease (GERD) undergo esophago-gastro-duodenoscopy (EGD), despite its low sensitivity in detecting reflux stigmata. Gastrin 17 (G-17) has been proposed to be related with GERD, due to the negative feedback between acid secretion and this hormone. We assessed the clinical usefulness of fasting G-17 serum determination for a non-invasive diagnosis of GERD in patients with typical symptoms.

Methods: We consecutively enrolled patients complaining of typical GERD symptoms in two different settings: a single referral center and a primary care setting. Control groups consisted of dyspeptic patients. All subjects underwent assessment of serum levels of G-17 and EGD.

Results: At the academic hospital, 100 GERD patients ( $\mathrm{n}=89$ with erosive esophagitis and 11 with Barrett's esophagus) had statistically significant low levels of G-17 as compared with 184 dyspeptic patients $(1.7 \pm 1.2$ $\mathrm{pg} / \mathrm{L}$ vs $8.9 \pm 5.7 \mathrm{pg} / \mathrm{L} \mathrm{p}<0.0001)$. Similarly, in the primary care setting, 163 GERD patients had statistically significant low levels of G-17 as compared with 132 dyspeptic patients $(0.5 \pm 0.2 \mathrm{pg} / \mathrm{L}$ vs. $4.0 \pm 2.6 \mathrm{pg} / \mathrm{L}$, $\mathrm{p}<0.0001$ ). Moreover, in the primary care setting, no statistically significant differences were found for $\mathrm{G}-17$ levels between patients with erosive and non-erosive reflux pattern ( $0.4 \pm 0.2$ vs $0.7 \pm 0.3 ; \mathrm{p}=0.08)$. In primary care, the accuracy of G-17 less than $1 \mathrm{pg} / \mathrm{L}$ to diagnose non-invasively GERD was $94.3 \%$.

Conclusions: Low levels of G-17 were detected in patients with erosive esophagitis and Barrett's esophagus in a referral center and in patients with typical GERD symptoms in a sample of patients from a primary care setting.

Key words: reflux disease - GERD - erosive esophagitis - Barrett's esophagus - gastrin 17 - pepsinogens

Abbreviations: BE: Barrett's esophagus; EGD: esophago-gastro-duodenoscopy; ERD: erosive reflux disease; G-17: gastrin 17; GERD: gastroesophageal reflux disease; $H$. pylori: Helicobacter pylori; LES: lower esophageal sphincter; LPR: laryngopharyngeal reflux; MII-pH: multichannel intraluminal impedance and $\mathrm{pH}$ monitoring; NERD: non-erosive reflux disease; NPV: negative predictive value; PG: pepsinogen; PPI: proton pump inhibitor; PPV: positive predictive value.

\section{INTRODUCTION}

Gastroesophageal reflux disease (GERD) is a complex entity with heterogeneous symptoms and complex pathogenesis that defies a simple diagnostic algorithm or categorical classification [1].

Gastroesophageal reflux disease is the most diagnosed digestive disorder in primary care [2-5]. Recent data showed that it has a prevalence ranging from 9.8-18\% in Europe to 18.1-
27.8\% in Northern America, with a lower incidence in Eastern Asia (2.5-7.8\%) [6].

The first line treatment for GERD patients with typical symptoms, such as heartburn and/or regurgitation, is proton pump inhibitor (PPI) therapy, that also presents a sensitivity and specificity of $71 \%$ and $44 \%$, respectively, in GERD diagnosis [7]. The addition of clinical history and disease-specific questionnaire do not always suffice to make a conclusive diagnosis of GERD [7]. In case of alarm features (eg, family history, weight loss, dysphagia, anemia and digestive bleeding), longstanding GERD symptoms and multiple risk factors (e.g. age $\geq 50$ years, white race, male sex, obesity) or in challenging cases, esophago-gastro-duodenoscopy (EGD) is required in order to evaluate the esophagus and the esophagogastric junction, and potentially to obtain biopsies from any 
lesion identified (e.g. areas of ectopic mucosa, strictures or mucosal abnormalities). However, low sensitivity limits the utility of this technique to diagnose GERD. Indeed, erosive reflux disease (ERD) or Barrett's esophagus (BE) occur in a minority of patients with GERD ( $<30 \%)$, whereas the majority of them are included in the non-erosive reflux disease (NERD) phenotype, characterized by typical reflux symptoms without any esophageal mucosal lesion visible at EGD [8]. In cases of atypical symptoms, the prevalence of reflux esophagitis is still more rare [9]. On the contrary, multichannel intraluminal impedance and $\mathrm{pH}$ monitoring (MII-pH) is considered the gold standard for reflux burden assessment. However, its low availability and invasiveness make it usable only in a small percentage of patients.

For all these reasons, especially in the primary care setting, GERD diagnosis remains based on clinical history, with the response to antisecretory therapy used as a confirmatory test. A non-invasive marker is still lacking, and the theoretically diagnostic algorithm based on EGD and pH-metry /MII-pH studies, is complex, expensive and time consuming.

Gastrin 17 (G-17) is a hormone produced $90 \%$ in the gastric antrum. It has been proposed as a non-invasive marker of GERD, due to the negative feedback between acid and this hormone [10]. Several studies have demonstrated the low levels of G-17 in patients with ERD and BE and various authors claimed that fasting G-17 levels could be a surrogate marker of high basal acid output, which predisposes to gastro-esophageal acid reflux $[11,12]$.

Thus, we aimed to assess the clinical usefulness of fasting G-17 serum determination for a non-invasive diagnosis of GERD in patients with typical symptoms, exploring its utility in two different settings, a referral center and a primary care setting.

\section{METHODS}

The study was carried out in two different settings: at a referral center and in a primary care setting in the NorthEastern of Italy. In the first case, we included all consecutive patients with typical GERD symptoms (heartburn and regurgitation) who underwent EGD from January to December 2018, at the endoscopic unit of the AltoVicentino Hospital (Veneto). All EGDs were performed by a single expert endoscopist. Biopsies were taken according to Sydney protocol and in case of any visible lesion and/or mucosa abnormality. Other causes of esophagitis were excluded (i.e. infections, eosinophilic esophagitis, medications). Patients were then classified, according to endoscopic and histological results as ERD, BE and NERD. Patients with NERD were excluded from the study group in the referral center. Erosive reflux disease was classified according to Los Angeles criteria and only grade B or superior were considered as diagnostic of GERD [7]. Barrett's esophagus was defined according to international criteria [13]. At the same time, consecutive patients complaining of dyspeptic symptoms (i.e. epigastric pain, nausea, post-prandial fullness, and early satiation) without endoscopic mucosal injuries were enrolled as the control group. All patients fulfilled the Rome IV criteria for functional dyspepsia diagnosis [14]. In addition, "bothersome epigastric burning" was not included in the criteria and patients also complaining of heartburn and/or regurgitation were not enrolled in the control group to avoid overlap (GERD-dyspepsia) and selection bias.

A second group of consecutive patients experiencing typical GERD symptoms (i.e. heartburn and/or regurgitation) was enrolled between January and December 2019, in a primary care setting. In parallel, consecutive patients with dyspeptic symptoms fulfilling the Rome IV criteria, but without heartburn and/or regurgitation or "bothersome epigastric burning", were enrolled as the control group. All patients underwent EGD with biopsies, as previously described, in the referral center. Dyspeptic patients with esophageal and/or gastric erosions and ulcer at EGD, were excluded.

In all patients enrolled in the study, Helicobacter pylori $(H$. pylori) infection, neoplasms, atrophic gastritis, autoimmune gastritis, chronic liver and renal diseases, ongoing PPI or H2 histamine receptor antagonist treatment, were considered exclusion criteria.

All patients performed a serological sample to measure pepsinogens (PGs) levels, G-17 and IgG against H. pylori by using GastroPanel (Elisa method: BioHit. Helsinki, Finland). Normal values are summarized as follows: PG I:30-160 mg/L, PG II: 2-15 mg/L, G-17: 1-10 pg/L, H. pylori IgG: <30 i.u.

The study was conducted according to the Good Clinical Practice and the Declaration of Helsinki for human studies. All patients gave their written informed consent for data collection and EGD.

Differences in proportions were compared using the chisquare or Fisher's exact test as appropriate. Unless otherwise specified, data are presented as median and range values. When data were not normally distributed, differences between groups were compared using Kruskal-Wallis and/or Mann-Whitney tests. A p-value of $<0.05$ was considered statistically significant. The Statistical Package for the Social Sciences (SPSS) version 16 software (SPSS Inc.; Chicago, IL, USA) was used for statistical analysis.

\section{RESULTS}

At the referral center, 256 patients complaining of typical GERD symptoms were firstly enrolled. After application of the exclusion criteria mentioned above and elimination of NERD patients, 89 consecutive subjects with ERD (51 males, mean age 52.0 \pm 9.0 years, range $31-76$ years) and 11 patients with histologically proven $\mathrm{BE}$ (9 males, mean age 55.6 \pm 4.0 , range 45-63) were included for further analysis. A sample of 248 patients with dyspeptic symptoms were initially identified and after applying exclusion criteria, 64 patients were excluded from the study, with 184 dyspeptic patients (98 males, mean age $49 \pm 8$ years, range $29-82$ years) finally included in the control group. The results obtained in GERD and dyspeptic patients were as follows: G-17 serum levels were significantly lower in both patients with $\mathrm{EE}$ and $\mathrm{BE}(1.7 \pm 1.2 \mathrm{pg} / \mathrm{L}$ and $1.6 \pm 0.9 \mathrm{pg} / \mathrm{L}$, respectively), compared to the control group $(8.9 \pm 5.7 \mathrm{pg} / \mathrm{L})(\mathrm{p}<0.0001)$. No significant differences were found between the studied groups for PG I (ERD: 87.3 \pm 8.2 $\mathrm{mg} / \mathrm{L}, \mathrm{BE}: 88.9 \pm 5.2 \mathrm{mg} / \mathrm{L}$, control group: $91.3 \pm 17.6, \mathrm{p}=0.9)$ and PG II (ERD: $6.4 \pm 5.1 \mathrm{mg} / \mathrm{L} ; \mathrm{BE}: 5.9 \pm 2.6 \mathrm{mg} / \mathrm{L}$, control group $=7.3 \pm 3.8, \mathrm{p}=0.19)$. 
In the primary care setting, initially 202 patients with GERD symptoms and 183 patients complaining of dyspeptic symptoms were included. After applying the above-mentioned exclusion criteria, 39 and 51 patients were ruled out from the GERD and control group, respectively. Thus, 163 patients with typical GERD symptoms ( 65 males, mean age $39.6 \pm 11.1$, range 18-84), of whom 57 shown erosive esophagitis at the endoscopy compared with 106 without erosions, and 132 consecutive dyspeptic patients (34 males, mean age $40.8 \pm 22.2$, range 18-79) were enrolled. Table I summarized the results obtained. Serum levels of G-17 resulted significantly lower in the GERD population $(0.5 \pm 0.2 \mathrm{pg} / \mathrm{L})$, compared with dyspeptic patients $(4.0 \pm 2.6 \mathrm{pg} / \mathrm{L})(\mathrm{p}<0.0001)$. No statistically significant differences were found for $\mathrm{G}-17$ levels between erosive and non-erosive esophagitis patients $(0.4 \pm 0.2$ vs $0.7 \pm 0.3 ; \mathrm{p}=0.08)$. No significant differences were observed between the studied groups regarding PG I $(84.8 \pm 37.7 \mathrm{mg} / \mathrm{L}$ in GERD patients vs. $79.4 \pm 26.8 \mathrm{mg} / \mathrm{L}$ in dyspeptic patients, $\mathrm{p}=0.8$ ) and PG II levels $(6.2 \pm 2.9 \mathrm{mg} / \mathrm{L}$ in GERD patients vs. $5.8 \pm 2.4 \mathrm{mg} / \mathrm{L}$ in dyspeptic patients, $\mathrm{p}=0.7$ )

Table I. Serological diagnosis of patients from the primary care cohort

\begin{tabular}{lccc}
\hline & $\begin{array}{c}\text { Dyspeptic patients } \\
(\mathrm{n}=132)\end{array}$ & $\begin{array}{c}\text { GERD } \\
(\mathrm{n}=163)\end{array}$ & $\mathrm{p}$ \\
\hline PG I (mg/L) & $79.4 \pm 26.8$ & $84.8 \pm 37.7$ & 0.167 \\
PG II (mg/L) & $5.8 \pm 2.4$ & $6.2 \pm 2.9$ & 0.205 \\
PG I/PG II & $14.5 \pm 4.3$ & $14.5 \pm 4.6$ & 1.000 \\
G-17 (pg/L) & $4.0 \pm 2.6$ & $0.5 \pm 0.2$ & 0.0001 \\
H. pylori. anti-bodies (i.u.) & $5.8 \pm 2.9$ & $4.9 \pm 2.1$ & 0.157 \\
\hline
\end{tabular}

GERD: gastroesophageal reflux disease; PG: pepsinogen; G-17: gastrin 17; H.pylori: Helicobacter pylori.

Table II summarized the overall results regarding the performance of blood levels of G-17 less than $1 \mathrm{pg} / \mathrm{L}$ in both referral center and primary care by comparing the GERD population and dyspeptic patients. Positive predictive values (PPV) results of $100 \%$ in both studied populations confirmed that low levels of G-17 are useful in identifying GERD patients.

Table II. Diagnostic performance of G-17 in studied populations

\begin{tabular}{lcccc}
\hline & \multicolumn{2}{c}{$\begin{array}{c}\text { Referral Center } \\
(\mathrm{n}=284)\end{array}$} & \multicolumn{2}{c}{$\begin{array}{c}\text { Primary Care } \\
(\mathrm{n}=295)\end{array}$} \\
\hline & $\begin{array}{c}\text { GERD } \\
(\mathrm{n}=100)\end{array}$ & $\begin{array}{c}\text { Controls } \\
(\mathrm{n}=184)\end{array}$ & $\begin{array}{c}\text { GERD } \\
(\mathrm{n}=163)\end{array}$ & $\begin{array}{c}\text { Controls } \\
(\mathrm{n}=132)\end{array}$ \\
\hline Gastrin 17 & 1.7 & 8.9 & 0.5 & 4.0 \\
(mean, range & $(0.2-3.1)$ & $(2.3-16.3)$ & $(0.1-0.9)$ & $(1.4-9.9)$ \\
Sensitivity & \multicolumn{2}{c}{$38 \%$} & \multicolumn{2}{c}{$62 \%$} \\
Specificity & \multicolumn{2}{c}{$100 \%$} & \multicolumn{2}{c}{$100 \%$} \\
PPV & \multicolumn{2}{c}{$100 \%$} & \multicolumn{2}{c}{$100 \%$} \\
NPV & $65.93 \%$ & \multicolumn{2}{c}{$96.8 \%$} \\
Accuracy & $71.82 \%$ & & $94.3 \%$ \\
\hline
\end{tabular}

Positive cut off test: G-17<1 pg/L; Negative cut off test: G-17>=1 pg/L; GERD: gastroesophageal reflux disease; PPV: positive predictive value; NPV: negative predictive value.

\section{DISCUSSION}

In the last years, the finding of low levels of G-17 has been find closely related with the endoscopic picture of esophagitis [15]. Thus, we investigated G-17 levels in a large population of subjects reporting reflux disease symptoms in comparison with a population of dyspeptic patients.

The role of gastrin in the pathophysiology of GERD is related to its feedback on the lower esophageal sphincter (LES) mediated by cholecystokinin receptors. It leads to a decrease in LES pressure and to an increase in the percentage of transient LES relaxation episodes associated with GERD [16]. The main role of G-17 consists of the stimulation of gastric acid secretion mediated by a specific receptor on parietal cells. Allescher et al. [10] analyzed the role of gastrin in the regulation of esophageal motility in healthy volunteers, showing that the infusion of G-17 caused an increase of LES pressure. Ninety percent of G-17 is secreted in the antrum, while gastrin 34 is produced in the gut. The first study on the use of G-17 as marker of GERD was published by Sipponen et al. [17] in 2005. A group of 199 dyspeptic patients was compared with 19 subjects affected by BE. Results confirmed that low levels of G-17 were related with the diagnosis of BE. Later, in 2008, Monkemuller et al. [18] published a matched case-control study, analyzing serum gastrin and PGs in relation to different grades of GERD severity. They found a significant difference among studied groups concerning PG I, but not for PG II, PG I/PG II ratio, H. pylori serology, nor gastrin levels. Pepsinogen I was lower in patients with NERD, and higher in patients with $\mathrm{BE}(\mathrm{p}=0.046)$. Pepsinogen I levels were also higher in $H$. pylori positive subjects. However, after adjusting for $H$. pylori status, the differences in PG I across patient groups were no longer statistically significant $(\mathrm{p}=0.298)$. Thus, the authors concluded that serum gastrin, PG I and II do not correlate with the different grades of severity of GERD. In contrast, Goni and et al. [15] reported a trend between G-17 levels and the severity of esophagitis (according to Los Angeles classification), with lower levels of the hormone being reported in cases of grade B esophagitis, intermediate levels in subjects with grade $\mathrm{A}$, and higher levels in NERD subjects. In 2009 Di Mario et al. [19] demonstrated that low levels of G-17 identify subjects with atypical symptoms of GERD such as chronic cough, non-cardiac chest pain, nocturnal apneas and non-allergic asthma. An interesting study conducted by Chourasia et al. [12] in 2011 evaluated GERD patients ( $n=123)$, who were not on acid-suppressive drugs and had not received anti- $H$. pylori therapy. All patients underwent EGD, tests for H. pylori detection, esophageal manometry, 24-h pH-metry, serum PG-I, PG-II and G-17 determination. Univariate and multivariate analyses were performed to assess independent predictors for ERD. The authors concluded that $H$. pylori infection was associated with lower acidic $\mathrm{pH}$ in stomach and less severe GERD symptoms. Low G-17, old age, hiatal hernia and the absence of $H$. pylori infection were the best predictors for ERD. In 2018, Miftahussurur et al. [20] performed a study on risk factors for GERD in Indonesia and found an association between PG I/II levels and GERD. A study performed in Japan by Ishimura et al. [21] investigated the increase in gastric acid secretion in healthy Japanese over two decades (from 1970's to 
1990's), by means of the determination of gastric acid secretion and the concentration of serum gastrin, PG I, PG II and $H$. pylori infection (GastroPanel).

Several studies have investigated the relationship between laryngopharyngeal reflux (LPR), that has been related to extraesophageal syndromes $[22,23]$, and pepsin secretion. In particular, Sone et al. [24] showed higher PGs concentration in elderly patients with LPR compared to controls. On the same topic, Luo et al. [25] demonstrated higher levels of both pepsin and PGs in subjects on-PPI treatment, in comparison with patients off therapy. Additionally, in patients on PPIs, the concentration of PG in middle ear effusion positively correlated with the intensity of PG protein expression in the cytoplasm of epithelial cells. The author concluded that pepsin and PG in middle ear effusion are probably caused by LPR and may be involved in its pathogenesis.

The present study, partially performed in a single referral center, confirmed the results of Sipponen et al. [17] and Chourasia et al. [12] on the relationship between low levels of serum G-17 and the findings of esophagitis in GERD population as well as the clinical manifestations of GERD (heartburn and/or regurgitation). In our study population in fact, G-17 levels less than 1pg/L were significantly lower in subjects with proven reflux disease, with ERD endoscopic documented, showing a very impressive PPV and specificity.

In the second part of the study, performed in a primary care setting, a large population of patients with upper gastrointestinal complaints was investigated, using both serology (GastroPanel) and EGD, with the aim to compare the serological findings with endoscopical picture. The results confirmed previous findings, with low levels of G-17 in GERD group compared to normal or increased concentrations in dyspeptic patients, always showing a high performance of both PPV and specificity and acceptable values for the negative predictive value (NPV), sensitivity and accuracy. In this study, both patients with ERD and NERD were analyzed together as the GERD population, with results that support the strong relationship between low levels of G-17 and clinical and endoscopic manifestation of GERD. With regard to pepsin secretion and the contribution of PG determination in the diagnosis of GERD, our experience was unable to find any difference between GERD and dyspeptic patients both in the referral center study and in the primary care setting.

Our work presents some limitations, mainly the lack of $\mathrm{pH}$-metry or MII-pH analysis to confirm GERD diagnosis in patients with typical GERD symptoms. It was not possible to discern patients with true NERD from those with reflux hypersensitivity and functional heartburn.

\section{CONCLUSIONS}

Our study confirmed the finding of a strong relationship between low levels of serum G17 and manifestations of GERD, with or without an endoscopic picture of esophagitis. Further investigations are required to confirm this finding in different settings of GERD, like those characterized by atypical symptoms, and to establish a relationship between G-17 values, reflux esophagitis severity and $\mathrm{pH}$ analysis results.

Conflicts of interest: None to declare.
Authors' contributions: F.D.M, E.S. designed the study, analyzed the data and wrote the manuscript. P.C., M.F., G.B., A.A., A.F., M.P.P., L.F., A.B., M.R., N.D.B: contributed to the conception of the study and to data analysis. K.R.C., M.G contributed to the conception of the work, data analysis and English language revision. All authors critically revised the manuscript, approved the final version to be published, and agree to be accountable for all aspects of the work.

\section{REFERENCES}

1. Savarino E, Bredenoord AJ, Fox M, et al; International Working Group for Disorders of Gastrointestinal Motility and Function. Advances in the physiological assessment and diagnosis of GERD. Nat Rev Gastroenterol Hepatol 2017;14:665-676. doi:10.1038/ nrgastro.2017.130

2. Dent J, El-Serag HB, Wallander MA, Johansson S. Epidemiology of gastro-oesophageal reflux disease: a systematic review. Gut 2005;54:710717. doi:10.1136/gut.2004.051821

3. Mahadeva S, Raman MC, Ford AC, et al. Gastro-oesophageal reflux is more prevalent in Western dyspeptics: A prospective comparison of British and South-East Asian patients with dyspepsia. Aliment Pharmacol Ther 2005;21:1483-1490. doi:10.1111/j.13652036.2005.02455.x

4. Wong WM, Lai KC, Lam KF, et al. Prevalence, clinical spectrum and health care utilization of gastro-oesophageal reflux disease in a Chinese population: a population-based study. Aliment Pharmacol Ther 2003;18:595-604. doi:10.1046/j.1365-2036.2003.01737.x

5. Kennedy T, Jones R. The prevalence of gastro-oesophageal reflux symptoms in a UK population and the consultation behaviour of patients with these symptoms. Aliment Pharmacol Ther 2000;14:15891594. doi:10.1046/j.1365-2036.2000.00884.x

6. El-Serag HB, Sweet S, Winchester CC, Dent J. Update on the epidemiology of gastro-oesophageal reflux disease: a systematic review. Gut 2014;63:871-880. doi:10.1136/gutjnl-2012-304269

7. Ghisa M, Barberio B, Savarino V, et al. The Lyon Consensus: Does It Differ From the Previous Ones? J Neurogastroenterol Motil 2020;26:311-321. doi:10.5056/jnm20046

8. Vakil N, van Zanten SV, Kahrilas P, Dent J, Jones R, Global Consensus Group. The Montreal definition and classification of gastroesophageal reflux disease: a global evidence-based consensus. Am J Gastroenterol 2006;101:1900-1920.

9. Vaezi MF. Atypical Manifestations of Gastroesophageal Reflux Disease. MedGenMed 2005;7:25.

10. Allescher HD, Stoschus B, Wünsch E, Schusdziarra V, Classen M. Effect of human gastrin-17 with and without acid suppression on human esophageal motility. Z Gastroenterol 1995;33:385-391.

11. Barchi A, Miraglia C, Violi A, et al. A non-invasive method for the diagnosis of upper GI diseases. Acta Biomed 2018;89:40-43. doi:10.23750/abm.v89i8-S.7917

12. Chourasia D, Misra A, Tripathi S, Krishnani N, Ghoshal UC. Patients with Helicobacter pylori infection have less severe gastroesophageal reflux disease: a study using endoscopy, 24-hour gastric and esophageal pH metry. Indian J Gastroenterol 2011;30:12-21. doi:10.1007/s12664010-0078-0

13. Weusten B, Bisschops R, Coron E, et al. Endoscopic management of Barrett's esophagus: European Society of Gastrointestinal Endoscopy [ESGE] Position Statement. Endoscopy 2017;49:191-198. doi:10.1055/s-0042-122140 
14. Stanghellini V, Chan FKL, Hasler WL, et al. Gastroduodena Disorders. Gastroenterology 2016;150:1380-1392. doi:10.1053/j. gastro.2016.02.011

15. Goni E, Riccò M, Franceschi M, et al. Gastrin 17 as non invasive marker of reflux disease Invasive marker of reflux disease. Dig Liver Dis 2015;47: e164-e165.

16. Straathof JW, Lamers CB, Masclee AA. Effect of gastrin-17 on lower esophageal sphincter characteristics in man. Dig Dis Sci 1997;42:25472551. doi: 10.1023/a:1018872814428

17. Sipponen P, Vauhkonen M, Helske T, Kaariainen I, Harkonen M. Low circulating levels of gastrin-17 in patients with Barrett's esophagus. World J Gastroenterol 2005;11:5988-5992. doi:10.3748/wjg.v11. i38.5988

18. Monkemuller K, Neumann H, Nocon M, et al. Serum gastrin and pepsinogens do not correlate with the different grades of severity of gastro-oesophageal reflux disease: a matched case-control study. Aliment Pharmacol Ther 2008;28:491-496. doi:10.1111/j.13652036.2008.03769.x

19. Di Mario F, Curlo M, Cavestro GM, et al. S1921 "GastroPanel Test" in the Clinical Outcome of GERD. Gastroenterology 2009;136(5 Suppl 1):A293. doi:10.1016/S0016-5085(09)61336-6
20. Miftahussurur M, Doohan D, Nusi IA, et al. Gastroesophageal reflux disease in an area with low Helicobacter pylori infection prevalence. PLoS One 2018;13:e0205644. doi:10.1371/journal.pone.0205644

21. Ishimura N, Owada $\mathrm{Y}$, Aimi $\mathrm{M}$, et al. No increase in gastric acid secretion in healthy Japanese over the past two decades. J Gastroenterol 2015;50:844-852. doi:10.1007/s00535-014-1027-y

22. Ghisa M, Coletta MD, Barbuscio I, et al. Updates in the field of non-esophageal gastroesophageal reflux disorder. Expert Rev Gastroenterol Hepatol 2019;13:827-838. doi:10.1080/17474124.201 9.1645593

23. Ghisa M, Marinelli C, Savarino V, Savarino E. Idiopathic pulmonary fibrosis and GERD: links and risks. Ther Clin Risk Manag 2019;15:10811093. doi:10.2147/TCRM.S184291

24. Sone M, Katayama N, Kato T, et al. Prevalence of Laryngopharyngeal Reflux Symptoms: Comparison between Health Checkup Examinees and Patients with Otitis Media. Otolaryngol Head Neck Surg 2012;146:562-566. doi:10.1177/0194599811434049

25. Luo HN, Yang QM, Sheng Y, et al. Role of pepsin and pepsinogen Linking laryngopharyngeal reflux with otitis media with effusion in children: Pepsinogen in LPR and OME. Laryngoscope 2014;124:e294e300. doi:10.1002/lary.24538 